\title{
On Social Experience, Prevention Consciousness and Network Fraud of College Students in Finance and Economics Based on Survey Data
}

\author{
Zejiong Zhou*, Li Han \\ School of Economics, Anhui University of Finance and Economics, Bengbu, Anhui, China \\ *aczzj123456@163.com
}

\begin{abstract}
With the development of the Internet, college students suffer from network fraud in many layers, which has aroused widespread concern in the society. Based on the questionnaire survey data of the current situation of college students suffering from online fraud in financial and economic colleges, this paper uses structural equation model to analyze the relationship between social experience, prevention awareness and college students suffering from online fraud. The study found that: (1) social experience not only has a significant direct effect on college students suffering from online fraud, but also has an indirect impact on college students suffering from online fraud through the pursuit of economic independence. (2) Prevention awareness has no significant direct effect on college students suffering from online fraud. Personal information protection plays a complete intermediary role between prevention awareness and college students suffering from online fraud. Based on the research results, it is proposed to strengthen the ideological and political education of college students, improve the awareness of risk prevention, build a long-term mechanism and link the comprehensive management of departments to prevent college students from suffering from online fraud and promote the healthy growth of college students.
\end{abstract}

Keywords: College students, Network fraud, Structural equation model.

\section{Introduction}

With the rapid development of Internet technology, the era of creating wealth with massive information has come. While enjoying a series of development achievements such as highquality communication technology, convenient and fast means of payment and rich and diverse online shopping platforms, people also have to face the new type of online fraud derived from the power of the Internet. Fraudsters use big data to obtain personal information through improper means, accurately lock users, and use people's psychological characteristics to formulate fraud routines, which makes it impossible to prevent. College Students' lack of social experience, weak awareness of risk prevention and lack of risk prevention ability make them the main target of fraudsters. In recent years, college students have suffered numerous cases of online fraud, and the injured students have suffered a serious blow both economically and psychologically.

Many domestic scholars have conducted in-depth research on the influencing factors of college students suffering from online fraud. On the one hand, college students are inexperienced and lack of social experience. Now college students generally belong to the Post- 00 generation, lack of social experience, lack of rationality and immature mind (Xiang Xinyi, 2020) [2]. Weak awareness of financial security and insufficient understanding of professional knowledge such as financial law (Lu Lingling, 2020) [3]. College students who lack careful thinking are difficult to resist temptation, so they are more likely to fall into the trap of fraud (Guo Yufang, 2020) [4]. Weak awareness of rights protection is also the main reason for the increase of online fraud (Chen Longtao, 2017) [5]. On the other hand, college students are the main users of the Internet, but their awareness of personal information protection is not strong, so they are more likely to become the target of fraudsters. Lu Huiteng is not confident in the Internet, and he is not likely to be cheated by others if he is exposed to the Internet (2019) [6]. If he is too confident in the Internet, he will be easily deceived by others if he is exposed to the Internet [6]. In addition, there are many loopholes in the information protection of college student management system, and the lack of fraud prevention education makes it difficult to eradicate online fraud (Li Wei, 2017) [8]. In addition, the immature psychology of college students is an important reason why they are deceived. College students are often compassionate, loyal, and willing to help students and friends in difficulty (GE Yiting, 2020) [9]. At the same time, they are also afraid, curious, competitive, profit seeking and lucky (Wu Rui, 2018) [10]. Criminals make full use of these psychological characteristics of college students to carry out relevant fraud activities and lure them into the hook (Chen Daijie, 2019) [11]. After being cheated, college students often dare not seek help from relatives, friends and the police because of their self-esteem and shame. They choose to be silent and have psychology such as "suffering losses is a blessing" and "spending money to buy lessons" (Wang Fei, 2018) [12].

Drawing on and referring to the research results of previous scholars on the influencing factors of college students suffering from online fraud, this paper analyzes and summarizes the following two hypotheses.

$\mathrm{H} 1$ : the lack of social experience of college students in financial and economic colleges makes them eager to achieve economic independence, which increases the possibility of suffering from network fraud, that is, "social experience $>$ pursuit of economic independence $>$ network fraud".

$\mathrm{H} 2$ : college students of finance and economics have weak awareness of network risk prevention, which leads to easy disclosure of personal information and increases the risk of network fraud, that is, "prevention awareness > information protection $>$ network fraud". 
Next, using the relevant data obtained from the questionnaire and structural equation model, this paper makes a quantitative analysis on the influencing factors of the frequent occurrence of online fraud among college students of finance and economics, tests hypothesis 1 and hypothesis 2 , and discusses how to reduce the occurrence of online fraud among college students of finance and economics and improve the ability of college students of finance and economics to prevent online fraud, put forward countermeasures and suggestions.

\section{Survey Object and Questionnaire Design}

\subsection{Survey Object}

A total of 927 valid questionnaires were collected by filling in the questionnaire online, covering more than 20 domestic financial and economic colleges and universities. Among them, there are 234 freshmen $(25.2 \%), 72$ sophomores $(7.7 \%)$, 405 junior students (43.6\%), 216 senior students $(23.3 \%)$, and the age range is between 18-24 years old.

\subsection{Questionnaire Design}

The questionnaire mainly includes two parts: basic information and influencing factor scale. The basic information mainly includes gender, grade, major, basic understanding of online fraud and how to choose after being cheated. It is used to understand the current situation of college students suffering from online fraud and make descriptive analysis according to the characteristics.

The scale mainly includes four scales: awareness of prevention, pursuit of economic independence, personal information protection and social experience. According to the scale, this paper constructs a structural equation model to analyze the relationship between social experience, prevention consciousness and college students' online fraud. (1) Social experience scale (SHJY): the SHJY scale contains 3 items, and each item adopts the 7-point scoring method [13]. Full agreement indicates that students are full of strong compassion, fear and fluke mentality, have relatively simple thoughts, and are most likely to fall into the trap of online fraud, with a score of 1 point; Completely disagree indicates that the students are very vigilant and have the lowest possibility of being subjected to online fraud, with a score of 7. (2) Prevention awareness scale (FFYS): the FFYS scale contains 3 items, and each item adopts the 7-point scoring method. Full agreement indicates that students are too confident in their own network risk prevention ability, have weak prevention awareness, and are more vulnerable to network fraud, with a score of 1; Totally disagree indicates that students have a strong awareness of network risk prevention and are the least likely to suffer from fraud, with a score of 7. (3) Pursuit of economic independence scale (JJDL): the JJDL scale contains 3 items, and each item adopts 7-point scoring method. Full agreement indicates that students have a strong desire to pursue economic independence and are more vulnerable to online fraud, with a score of 1; Completely disagree indicates that students do not have the desire to pursue economic independence, and have the lowest chance of encountering online fraud, with a score of 7. (4) Personal information protection scale $(\mathrm{XXBH})$ : the $\mathrm{XXBH}$ scale contains 3 items, and each item adopts the 7-point scoring method. If $0 \%$ is achieved, it indicates that students ignore personal information protection in life and are very easy to disclose personal information and suffer from online fraud, 1 point is assigned; $100 \%$ indicates that students pay great attention to personal information protection in life and are the least likely to suffer from online fraud. A score of 7 points is assigned.

\section{Investigation Results and Analysis}

\subsection{Descriptive Analysis on the Current Situation if College Students Suffering from Online Fraud}

By sorting out the collected effective questionnaires and using SPSS software, this paper makes a descriptive analysis on the current situation of Internet fraud suffered by college students in financial and economic colleges, and obtains the following results.

Among the college students of Finance and economics, $42.7 \%$ encountered online fraud but did not lose money, $32.9 \%$ encountered online fraud and lost money, and $22.3 \%$ did not encounter online fraud. In terms of the amount of fraud, most of the amount of fraud is less than 3000 yuan $(72.1 \%)$, but there are also cases where the amount of fraud is more than 10000 yuan $(5.4 \%)$. The most common forms of online fraud were swindling $(82.5 \%)$, telephone fraud $(78.6 \%)$ and social software fraud (62.1\%). Online fraud information mainly comes from QQ friends or QQ groups (83.5\%), SMS (73.8\%) and email (33.4\%).

According to sex, female college students $(72.3 \%)$ are more likely to be cheated than male college students $(27.7 \%)$. According to the grade, there are more people suffering from online fraud in Junior (36.1\%) and senior (41.6\%) than freshmen (19.4\%) and sophomores (2.7\%).

\subsection{Structural Equation Model Analysis of Influencing Factors of College Students Suffering from Online Fraud}

According to Xinshibo's research, structural equation model is an important tool for analyzing influencing factors and constructing rating index system [14]. Therefore, structural equation model is used to analyze the influencing factors of College Students' frequent online fraud and verify hypothesis 1 and hypothesis 2 .

\subsubsection{Construct Facets and Verify}

AMOS software is used to construct five dimensions: personal information protection $(\mathrm{XXBH})$, prevention awareness (FFYS), pursuit of economic independence (KKDL), social experience (SHJY) and online fraud (WLZP), and the survey data are brought into the corresponding dimensions for reliability and validity test. According to MacKinnon's research theory, the acceptable level of questionnaire reliability is 0.36 , the factor load needs to reach 0.6 , and there should be significant differences between dimensions. Sort out the calculated inspection results. The specific results are shown in Table 1 and table 2. 
Table 1. Reliability test of questionnaire

\begin{tabular}{|c|c|c|c|c|c|c|c|c|}
\hline & & UNSTD. & S.E. & t-value & STD. & SMC & C.R. & AVE \\
\hline \multirow{5}{*}{$\begin{array}{l}\text { Personal information } \\
\text { protection }\end{array}$} & ХХВН2 & 1.000 & & & .700 & .490 & .837 & .507 \\
\hline & XXBH7 & 1.017 & .089 & 11.471 & .766 & .587 & & \\
\hline & XXBH8 & 1.062 & .097 & 10.980 & .724 & .524 & & \\
\hline & XXBH3 & 1.064 & .098 & 10.907 & .718 & .516 & & \\
\hline & XXBH1 & .912 & .091 & 9.975 & .648 & .420 & & \\
\hline \multirow[t]{3}{*}{ Awareness of prevention } & MUZX1 & 1.000 & & & .755 & .570 & .829 & .618 \\
\hline & MUZX2 & 1.224 & .099 & 12.301 & .860 & .740 & & \\
\hline & MUZX4 & 1.142 & .096 & 11.960 & .738 & .545 & & \\
\hline \multirow[t]{3}{*}{ Social experience } & SHJY1 & 1.000 & & & .889 & .790 & .880 & .711 \\
\hline & SHJY2 & .994 & .055 & 18.202 & .905 & .819 & & \\
\hline & SHJY7 & .839 & .057 & 14.654 & .724 & .524 & & \\
\hline \multirow[t]{3}{*}{$\begin{array}{l}\text { Pursue economic } \\
\text { independence }\end{array}$} & JJDL2 & 1.000 & & & .893 & .797 & .920 & .794 \\
\hline & JJDL4 & 1.147 & .048 & 23.863 & .943 & .889 & & \\
\hline & JJDL5 & 1.008 & .050 & 19.976 & .835 & .697 & & \\
\hline \multirow[t]{3}{*}{ Network fraud } & zp3 & 1.000 & & & .405 & .164 & .373 & .410 \\
\hline & zp4 & -2.165 & .508 & -4.261 & -.865 & .748 & & \\
\hline & zp5 & -1.616 & .286 & -5.650 & -.565 & .319 & & \\
\hline
\end{tabular}

Table 2. Differential validity table

\begin{tabular}{ccccccc}
\hline & AVE & JJDL & FFYS & WLZP & SHJY & XXBH \\
\hline JJDL & .794 & .891 & & & & \\
FFYS & .618 & .472 & .786 & & & \\
WLZP & .410 & -.141 & -.064 & .640 & .843 & \\
SHJY & .711 & .581 & .395 & .018 & .740 & .712 \\
XXBH & .507 & -.546 & -.591 & .075 & -.740 \\
\hline
\end{tabular}

According to the $\mathrm{p}$ value corresponding to t-value in column 5 of Table 1, the $\mathrm{P}$ values are three stars, significant $(<0.001)$, and each item passes the t-test. Column 7 "factor load (STD)" is basically greater than 0.6; Column 8 "item reliability (SMC)" is basically greater than 0.36; Column 9 "C.R." except for the C.R. value of network fraud is 0.373 , the C.R. value of other dimensions is above 0.8; Column 10 "convergence reliability (AVE)" is basically greater than 0.5 . Comprehensive analysis of the above indicators shows that the reliability of the questionnaire is good. The diagonal numbers in Table 2 are AVE1/2, and each number is significantly larger than the numbers on the left and below. It can be seen that the questionnaire has passed the differential validity test.

\subsubsection{Model Establishment and Model Matching Test}

After the questionnaire passes the reliability and validity test, the model construction and SEM matching test will be carried out. According to the research theory of Jackson Dennis L, the main indicators of SEM model matching include CMIN/DF, GFI, AGFI, CFI, RMSEA, etc. Where CMIN/DF $<3$, GFI, AGFI, CFI need to be greater than 0.9 and RMSEA less than 0.08 . Therefore, the structural equation model of college students suffering from online fraud is established based on the above five aspects, and the model fit in Amos is used to test the model.

According to the calculation results, the chi square of the model is 493.470 , the degree of freedom (df) is 112 , and the model chi square degree of freedom ratio (CMIN/DF) is 4.406; The fitness index (GFI) of the model is about 0.9 , which is close to 1 , indicating that the structural equation model of college students suffering from online fraud has a good fit; The adjusted model fitness index (AGFI) is about 0.8, indicating that after eliminating the influence of degrees of freedom on GFI, the fitting degree of structural equation of college students suffering from online fraud is still acceptable; The comparative fit index (CFI) of the model is about 0.9, indicating that the fitting degree of the hypothetical theoretical model to be tested is better than that of the independent model. The mean square sum of squares (RMSEA) of the progressive residuals of the model is 0.105 , indicating that the structural equation model of college students suffering from online fraud can fit the actual data. From the above index test results, the fitting effect of structural equation model of college students suffering from online fraud is basically acceptable.

\subsubsection{Intermediary Effect Test}

After the model passes the SEM matching test, in order to further study the relationship between social experience, prevention consciousness and college students suffering from online fraud, that is, to test hypothesis 1 and hypothesis 2, we need to test the intermediary effect of the model. According to Bradley Efron's research theory, the deviation correction nonparametric percentile Bootstrap is used for testing, 2000 repeated samples are set, and the $95 \%$ confidence interval is calculated. When the confidence interval of bias corrected estimation method and percentile estimation method does not contain 0 , it shows that the mediation effect is significant through the test, otherwise the mediation effect is not significant.

Based on the questionnaire data and using the indirect, direct \& total effects test function and deviation correction nonparametric percentile Bootstrap function in AMOS software, the intermediary effect test is carried out. The specific test results are shown in Table 3. 
Table 3. Bootstrap analysis of significance test of mediating effect and its effect

\begin{tabular}{cccccccc}
\hline \multirow{2}{*}{ SIE } & \multirow{2}{*}{ point estimation } & \multicolumn{2}{c}{ product of coefficient } & \multicolumn{2}{c}{ bias-corrected } & \multicolumn{2}{c}{ percentile } \\
\cline { 3 - 8 } & & SE & Z & Lower & Upper & Lower & Upper \\
\hline FFYS > XXBH>WLZP & -.023 & .012 & -1.917 & -.050 & -.008 & -.046 & -.005 \\
SHJY > JJDL > WLZP & -.060 & .032 & -1.875 & -.124 & -.017 & -.117 & -.013 \\
SHJY>WLZP & .065 & .032 & 2.031 & .021 & .127 & .017 & .123 \\
FFYS > WLZP & .005 & .050 & .100 & -.079 & .083 & -.081 & .082 \\
lj1 v slj2 & .037 & .036 & 1.028 & -.014 & .106 & -.018 & .100 \\
\hline
\end{tabular}

It can be seen from table 3 that the confidence intervals corresponding to $\mathrm{H} 1(-0.124,-0.017)$ and $\mathrm{H} 2$ (- 0.050, 0.008 ) do not contain 0 , indicating that the mediation effect of these two paths is significant. According to the conclusion of the intermediary model proposed by Wen Zhonglin, the confidence interval corresponding to the "social experience $>$ online fraud" path does not contain 0 , indicating that the direct effect of social experience on online fraud is significant, and it is part of the intermediary effect. The confidence interval corresponding to the path of "prevention awareness $>$ network fraud" contains 0 , indicating that the effect of prevention awareness on network fraud is not significant, indicating that this intermediary effect is a complete intermediary effect [15].

\subsubsection{Discuss}

The results of this study found that "social experience $>$ pursuit of economic independence $>$ online fraud" is part of the intermediary effect, that is, social experience not only has a significant direct effect on college students suffering from online fraud, but also has an indirect impact on college students suffering from online fraud through the pursuit of economic independence, college students' pursuit of economic independence plays a partial intermediary role between social experience and college students' online fraud. Next, the direct effect and indirect effect are further analyzed. First of all, the direct effect is significant, and the direct effect value is 0.07 and the effect amount is $75.92 \%$, indicating that college students' lack of social experience leads to an increase in their possibility of suffering from online fraud, which is consistent with the research conclusions of Lu Lingling [3], Guo Yufang [4], Chen Longtao [5]. Because college students are inexperienced and do not understand the complexity of society and interpersonal relationships, they are more likely to trust others, which has become the target of online fraud. Secondly, the indirect effect is significant. The intermediary path effect value is -0.022 and the effect amount is $23.86 \%$, indicating that college students lack social experience, which leads them to think it is easier to make money and are keen on pursuing economic independence and realizing economic freedom, so they are easy to be deceived and fall into the trap of online fraudsters, increasing the possibility of being cheated by online frauds, This is consistent with the research conclusions of Chen Daijie [11] and others. Thus hypothesis 1 is proved.

"Prevention awareness $>$ information protection $>$ network fraud" is a complete intermediary effect, that is, college students' prevention awareness has no direct impact on suffering from network fraud, but will indirectly affect college students' suffering from network fraud by affecting personal information protection, and college students' personal information protection plays a complete intermediary role between College Students' prevention awareness and suffering from network fraud. College students are an important group of Internet users. Long-term contact with and use of the Internet will make college students too confident in their risk prevention awareness and ability. They think they are very familiar with the Internet, Internet Finance and other projects, and can immediately identify and avoid online fraud. This blind confidence in their own prevention awareness and ability leads to the inadequate protection of College Students' personal information, which increases the possibility of suffering from network fraud. This is consistent with the research conclusions of Shen Huizhang [6], Lv Teng [7], Li Wei [8]. This is supported by hypothesis 2 .

\section{Conclusions and Suggestions}

This paper discusses the relationship between college students' financial fraud awareness and social experience. The results show that social experience not only has a significant direct effect on college students suffering from online fraud, but also has an indirect impact on college students suffering from online fraud through the pursuit of economic independence; Prevention awareness has no significant direct effect on college students suffering from online fraud. Personal information protection plays a complete intermediary role between prevention awareness and college students suffering from online fraud. Due to their inexperience and lack of social experience, college students have fluke psychology and profit seeking psychology; Weak awareness of prevention and inadequate protection of personal information are important reasons for the frequent occurrence of college students' online fraud. According to the research results and actual situation, the following suggestions are put forward:

(1) Strengthen the ideological and political education of college students and improve the awareness of risk prevention. Correct the thinking of college students, enhance their fraud prevention awareness and improve their risk prevention ability are the most fundamental solutions. First, colleges and universities should strengthen ideological and political education, teach college students to resist temptation, not greedy for petty profits and blind comparison, and do their own work well. Second, schools and teachers should implement the publicity of online fraud knowledge and cases, and enhance students' awareness of fraud prevention.

(2) Build a psychological defense line against deception and deal with deception rationally and sensitively. The key to reducing college students' online fraud lies in college students themselves. College students themselves should build a psychological defense line against fraud, be rational and smart in the face of fraud, and dare to protect their rights in the face of fraud. First, we should take every campus anti fraud propaganda seriously, timely understand and learn new online fraud knowledge and cases, improve risk prevention ability, and see through online fraud at the first time. Second, resolutely resist temptation, do not compete, pursue profits and seek novelty, and be vigilant against the urgent need of 
"relatives and friends" to borrow money. Third, after suffering from online fraud, we should adjust our psychology, actively seek the help of teachers, parents and police, take up the weapon of law and safeguard our legitimate rights and interests.

(3) Build a long-term mechanism and link the comprehensive management of departments. Combating telecom network fraud requires the joint efforts and comprehensive management of all departments. First, the legislature should strengthen the legislative investigation of telecom network fraud, timely supplement the use norms and illegal legal systems such as the opening of relevant bank cards, the handling of telephone cards and the registration of social software accounts, so as to achieve the matching of relevant laws and regulations to combat network fraud, so that there are laws to follow. Second, the public security department should fully carry out the fight against online fraud, strengthen cooperation with the judicial department of the school, financial and communication departments, and comprehensively deal with online fraud.

\section{References}

[1] Lou Yongtao, Tang Xiang. Reflection on the prevention and control of telecom network fraud in the era of big data [J]. Journal of Chongqing University of Technology (SOCIAL SCIENCES), 2020, 34 (03): 121-128.

[2] Xiang Xinyi. Research on College Students' online fraud from the perspective of personal information security $[\mathrm{J}]$. Journal of Harbin vocational and technical college, 2020 (03): 101-103.

[3] Lu Lingling. Research on financial security risk prevention strategies of College Students under the background of Internet [J]. Journal of Jining Normal University, 2020,42 (05): 19-22.

[4] Guo Yufang, Wang Li. Analysis of College Students' awareness of network risk prevention after 00 -- Reflection on social problems such as "telecom network fraud" [J]. Journal of Hanjiang Normal University, 2020, 40 (02): 114-118.

[5] Chen Longtao, Zhang Yuwei, Sun Yihang. Research on the prevention of College Students' online fraud from the perspective of network security [J]. Journal of Kunming University of Technology (SOCIAL SCIENCE EDITION), 2017, 17 (05): 86-91.

[6] Shen Huizhang, Yuan Xingchen. Communication network fraud cases targeting college students and their investigation and prevention [J]. Journal of Guangxi Police College, 2019,32 (01): 37-42.

[7] Lv Teng. Causes and preventive measures of college students being cheated by Internet [J]. Western leather, 2016,38 (20): 252 .

[8] Li Wei, Li Lei. On the risk prevention mechanism of "telecom network fraud" in Colleges and Universities under the new normal [J]. Journal of Jilin Normal University of engineering and technology, 2017, 33 (07): 8-11.

[9] Ge Yiting, Wang Panpan, Kessel Kurban. Psychological analysis and coping strategies of college students cheated by means of network fraud [J]. Psychological monthly, 2020, 15 (21): 30-31.

[10] Wu Rui. Causes and Countermeasures of college students suffering from network fraud [J]. Legal system Expo, 2018 (33): 201-202.

[11] Chen Daijie. The psychological reasons for the high incidence of fraud cases in Colleges and Universities under the background of Internet plus [J]. Economic Research Guide, 2019 (30): 198-199.

[12] Wang Fei, Wang Tingting, Mo Bin, Huang Siyu, Lu Kun, Zhang Ling. Study on the psychological changes of college students cheated by telecom network fraud [J]. E-commerce, 2018 (11): 94-96.

[13] Cordano M. An Introduction to the Basics of Structural Equation Modeling (SEM) [J].1999,10(07):965-976.

[14] Xin Shibo, Chen Yan, Zhang Chen. Summary of applied research results of structural equation model theory $[\mathrm{J}]$. Industrial technology and economy, 2014, 33 (05): 61-71.

[15] Wen Zhonglin Zhang Lei, Hou Jietai, Liu Hongyun. Test of Mediation Program and its application [J]. Journal of psychology, 2004 (05): 614-620. 